\title{
Rechtsprechung
}

\section{Einbeziehung eines von einem Auktionshaus berechneten Aufgelds in den Zollwert des ersteigerten Objekts}

Bundesfinanzhof, Beschluss vom 21. September 2011 - VII R 25/10

Ein von einem Auktionshaus dem Ersteigerer neben dem Kaufpreis in Rechnung gestelltes Aufgeld ist keine Einkaufsprovision, wenn es jedem Ersteigerer unabhängig von einer ihm gegenüber erbrachten Leistung des Auktionshauses berechnet wird.

\section{Gründe}

- I. Der Kläger und Revisionskläger (Kläger) ersteigerte als sog. Fernbieter ein Bild bei einem Schweizer Auktionshaus. Neben dem Zuschlagspreis in Höhe von 1.300 CHF hatte er ein vom Auktionshaus berechnetes Aufgeld von $260 \mathrm{CHF}$ zuzüglich 7,6 \% Schweizer Umsatzsteuer, insgesamt also 1.579,75 CHF zu zahlen. Dieser Rechnungspreis wurde bei der Einfuhrabfertigung des in das Zollgebiet der Gemeinschaft verbrachten Bildes der Festsetzung der Einfuhrumsatzsteuer zugrunde gelegt. Den Antrag des Klägers, bei der Abgabenfestsetzung lediglich den Zuschlagspreis ohne Aufgeld des Auktionshauses zu berücksichtigen, sah der Beklagte und Revisionsbeklagte (das Hauptzollamt - HZA -) als Antrag auf teilweise Erstattung der Einfuhrumsatzsteuer an, den das HZA ablehnte.

Die hiergegen nach erfolglosem Einspruchsverfahren erhobene Klage wies das Finanzgericht (FG) ab. Das FG urteilte, es handele sich bei dem an das Auktionshaus zu zahlenden Aufgeld um keine in den Zollwert gemäß Art. 32 Abs. 1 Buchst. a Ziff. i des Zollkodex (ZK) bzw. Art. 33 Buchst. e ZK nicht einzubeziehende Einkaufsprovision. Es sei nach den Auktionsbedingungen nicht erkennbar, dass das Aufgeld für eine dem Ersteigerer in dessen besonderem Auftrag zu erbringende Leistung gezahlt werde. Vielmehr sei das Aufgeld von jedem Ersteigerer zu bezahlen, unabhängig davon, ob er dem Auktionshaus einen Auftrag, für ihn zu bieten, erteilt oder persönlich bei der Auktion mitgeboten habe.

Mit seiner Revision macht der Kläger geltend, das dem Auktionshaus gezahlte Aufgeld sei eine Einkaufsprovision. Mit der Abgabe eines Gebots erteile der Bieter dem Auktionshaus den Auftrag, für inn unter Beachtung der Auktionsregeln den Zuschlag zu erteilen. Das Auktionsaufgeld werde daher dem Auktionshaus dafür gezahlt, dass dieses für den Käufer i.S. des Art. 32 Abs. 4 ZK beim Kauf der zu bewertenden Ware tätig werde. Dies gelte jedenfalls dann, wenn das Auktionshaus - wie im Streitfall - aufgrund eines vorab erteilten Auktionsauftrags für einen Bieter tätig werde und mitbiete.
II. Die Entscheidung ergeht gemäß § 126 a der Finanzgerichtsordnung (FGO). Der Senat hält einstimmig die Revision für unbegründet und eine mündliche Verhandlung nicht für erforderlich. Die Beteiligten sind davon unterrichtet worden und hatten Gelegenheit zur Stellungnahme.

Das FG hat die Klage zu Recht abgewiesen. Der die teilweise Erstattung der Einfuhrumsatzsteuer versagende Bescheid vom 17. Januar 2006 ist rechtmäßig (§ 101 Satz 1 FGO).

Der Kläger hat keinen Anspruch auf Erstattung des Teils der Einfuhrumsatzsteuer, der auf das vom Auktionshaus in Rechnung gestellte Aufgeld entfällt. Die unter Zugrundelegung des gesamten Rechnungsbetrags in Höhe von 1.579,75 CHF festgesetzte Einfuhrumsatzsteuer ist der i.S. des § 21 Abs. 2 des Umsatzsteuergesetzes (UStG) iVm Art. 236 ZK gesetzlich geschuldete Betrag.

Nach $\S 11$ Abs. 1 UStG wird der Umsatz für die Festsetzung der Einfuhrumsatzsteuer nach dem Wert des eingeführten Gegenstands nach den jeweiligen Vorschriften über den Zollwert bemessen. Der Zollwert eingeführter Waren ist nach Art. 29 Abs. 1 ZK grundsätzlich ihr Transaktionswert, d.h. der für die Waren bei einem Verkauf zur Ausfuhr in das Zollgebiet der Union tatsächlich gezahlte oder zu zahlende Preis. Da der Kläger für das in das Zollgebiet der Union eingeführte Bild einen Preis von $1.579,75$ CHF (= 1.019,79 EUR ) zu zahlen hatte, ist dieser Betrag der Transaktionswert, welcher der Bemessung der Einfuhrumsatzsteuer zugrunde zu legen ist.

Nach vorgenannter Vorschrift ist der Transaktionswert zwar ggf. gemäß Art. 32 und 33 ZK zu berichtigen; die Voraussetzungen der im Streitfall allein in Betracht kommenden Vorschrift des Art. 33 Buchst. e ZK, derzufolge Einkaufsprovisionen, sofern sie getrennt vom tatsächlich gezahlten oder zu zahlenden Preis ausgewiesen werden, nicht in den Zollwert einbezogen werden, liegen jedoch nicht vor.

Zwar ist mit der Rechtsprechung des Gerichtshofs der Europäischen Union (EuGH) davon auszugehen, dass ein Abzugsposten i.S. des Art. 33 ZK, der in der Einfuhrzollanmeldung nicht getrennt vom Kaufpreis der Waren ausgewiesen ist, gleichwohl 
im Rahmen einer nachträglichen Überprüfung der Zollanmeldung als nicht in den Zollwert einzubeziehen geltend gemacht und unter den Voraussetzungen des Art. 236 ZK eine Erstattung bereits entrichteter Einfuhrabgaben beansprucht werden kann (vgl. EuGH-Urteile vom 20. Oktober 2005 - C-468/03 - Overland Footwear -, Slg. 2005, I-8937, Zeitschrift für Zölle und Verbrauchsteuern - ZfZ - 2005, 408; vom 15. Juli 2010 - C-354/09 - Gaston Schul -, ZfZ 2010, 238; Müller-Eiselt EG-Zollrecht, Zollkodex/Zollwert, Fach 4233 Rz 80 ff.; Krüger in: Dorsch, Zollrecht, Art. 33 ZK $\mathrm{Rz} 29$ ). Zu Recht hat jedoch das FG im Streitfall geurteilt, dass es sich bei dem an das Auktionshaus zu zahlenden Aufgeld nicht um eine Einkaufsprovision handelt.

Unter einer Einkaufsprovision ist nach Art. 32 Abs. 4 ZK ein Betrag zu verstehen, den ein Einführer jemandem dafür zahlt, dass er für ihn beim Kauf der zu bewertenden Ware tätig wird, wobei es nach der Rechtsprechung des erkennenden Senats nicht darauf ankommt, ob der Einkaufsagent als unmittelbarer oder mittelbarer Stellvertreter des Käufers tätig wird; entscheidend ist allein, dass er bei dem Geschäft für Rechnung seines Auftraggebers, des Käufers in der Gemeinschaft, handelt (Senatsurteil vom 4. November 1999 - VII R 43/98, BFHE 190, 514, ZfZ 2000, 121).

Ein Auktionshaus und sein Auktionator werden jedoch für den Verkäufer einer zu versteigernden Ware tätig. Dies ergibt sich im Streitfall auch aus den (vom FG in Bezug genommenen) Auktionsbedingungen des Schweizer Auktionshauses, wonach von diesem die Objekte im Namen und für Rechnung Dritter verkauft werden, wofür es vom Einlieferer eine Kommission erhält. Ein Auktionshaus hat vertragliche Beziehungen zum Verkäufer und erbringt ihm gegenüber seine Leistungen wie z.B. Übernahme und Aufbewahrung des zu versteigernden Gegenstands, Aufnahme in den Versteigerungskatalog, Präsentation bei der Versteigerung usw. Auch mit der Verwahrung des vom Meistbietenden ersteigerten Gegenstands durch das Auktionshaus vom Zuschlag bis zur Abholung sowie der Bereitstellung einer transportsicheren Verpackung, auf die der Kläger zur Begründung seiner Ansicht verweist, erfüllt das Auktionshaus vertragliche Nebenpflichten des Verkäufers und nicht etwa Pflichten aus einem mit einem potenziellen Käufer geschlossenen Kommissionsvertrag.

Käuferaufgaben nimmt ein Auktionshaus bei einer Versteigerung hingegen nicht wahr, da es lediglich die Gebote potenzieller Käufer für den Verkäufer entgegennimmt und in dessen Auftrag dem Meistbietenden den Zuschlag erteilt. Für den Käufer wird ein Auktionshaus bei einer Versteigerung nur dann tätig, wenn es - wie es nach den Angaben des Klägers in seinem Fall gewesen ist - von einem Kaufinteressenten den Auftrag erhalten hat, für diesen mitzusteigern und damit auch für dessen Rechnung tätig zu werden. Erhält es in einem solchen Fall für dieses Mitsteigern von demjenigen, dem der Zuschlag erteilt und der damit Käufer der Ware wird, eine Provision, wird man diese zwar als eine Einkaufsprovision i.S. des Art. 32 Abs. 4 ZK ansehen können. Jedoch verhielt es sich im Streitfall nicht so, weil nach den Feststellungen des $F G$, an die der Senat mangels zulässiger und begründeter Revisionsgründe gebunden ist ( $\$ 118$ Abs. 2 FGO), das Aufgeld nach den Auktionsbedingungen von jedem Ersteigerer zu bezahlen war, unabhängig davon, ob dieser bei der Versteigerung selbst anwesend war und geboten hatte oder als Fernbieter dem Auktionshaus einen Auftrag zum Mitbieten erteilt hatte. Als ein Entgelt für das Mitbieten im Auftrag des Klägers kann das im Streitfall berechnete Aufgeld daher nicht angesehen werden.

Das Aufgeld wäre im Übrigen auch dann bei der Zollwertermittlung zu berücksichtigen, wenn man der Ansicht des Klägers folgte, das Auktionshaus sei einem Makler vergleichbar, der einen Kaufvertrag vermittelt (wofür nach Ansicht des Senats allerdings nichts spricht), da Maklerlöhne - anders als Einkaufsprovisionen - gemäß Art. 32 Abs. 1 Buchst. a Ziff. i ZK dem tatsächlich gezahlten oder zu zahlenden Preis hinzuzurechnen sind und somit zum Zollwert gehören.

Soweit der EuGH entschieden hat, dass bei der Versteigerung von Kunstgegenständen, Sammlungsstücken und Antiquitäten im Zollgebiet der Gemeinschaft, die im Verfahren der vorübergehenden Verwendung eingeführt wurden, der ermäßigte Umsatzsteuersatz nicht auch auf die dem Auktionator gezahlte Provision anzuwenden ist (EuGH-Urteil vom 9. Februar 2006 C-305/03 - Kommission/Vereinigtes Königreich -, Slg. 2006, I-1213), lässt sich hieraus - wie das FG zutreffend ausgeführt hat - für die im Streitfall zu treffende zollwertrechtliche Entscheidung, ob das dem Schweizer Auktionshaus zu zahlende Aufgeld eine Einkaufsprovision war, nichts herleiten. (Entscheidung von der Redaktion bearbeitet.) 